
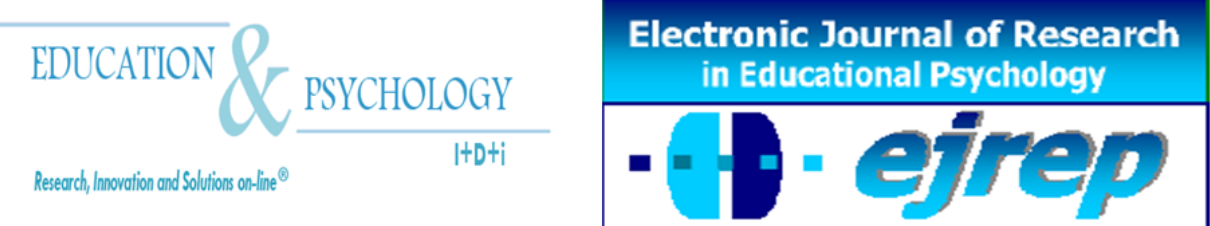

Editorial EOS

\title{
Evaluación de un programa educativo de manejo conductual
}

\section{Antonio Coronado Hijón}

Jefe Dpto. de Orientación, I.E.S. Joaquín Turina, Sevilla

Dpto. de Ciencias Sociales, Universidad Pablo de Olavide, Sevilla

\section{España}

Antonio Coronado Hijón, Facultad de Ciencias Sociales, Universidad Pablo de Olavide, Ctra. De Utrera, Km 1, 41013 Sevilla, España. E-mail: acoronado@upo.es

(C) Education \& Psychology I+D+i and Editorial EOS (Spain) 


\section{Resumen}

Introducción. En el presente estudio, se expone la aplicación de un programa de manejo de contingencias para la mejora del comportamiento en un aula de Educación Secundaria obligatoria, donde la mayoría del alumnado presentaba un comportamiento disruptivo.

Método. Para este estudio se aplicó un tratamiento conductual denominado "Tiempo de buen comportamiento", basado en un sistema de "economía de fichas" orientado hacia el grupo con contingencias interdependientes, adaptado en parte de técnicas parecidas como el "Juego del Buen Comportamiento" (Good behavior Game, GBG) y la técnica del "reloj de buena conducta".

Resultados. Los resultados en "“Tiempo de Buena Conducta” obtenidos, muestran claramente una importante mejora que lleva a alcanzar el $90 \%$ del tiempo de las sesiones lectivas de 50 minutos, desde el 50\% aproximado de la línea base.

Discusión y Conclusiones. Los resultados de este estudio muestran la eficacia de la técnica de contigencia con grupos "Tiempo de Buena Conducta" (Good Behavior Time, GBT) en la reducción de comportamientos disruptivos en contextos educativos. Las cualidades de esta técnica son la facilidad de su funcionamiento, tanto para el alumnado como para el profesorado, su bajo coste económico así como su adaptación a las características de cada grupo, aspectos que conforman el resultado de eficacia deseada.

Palabras Clave: técnicas de contingencias grupales, tiempo de buen comportamiento, juego del buen comportamiento, comportamiento disruptivo, convivencia en el aula, economía de fichas, reloj de buena conducta.

Recibido: 01/10/08 Aceptación inicial: 14/10/08ａceptación final: 24/02/09 


\title{
Assessment of an educational behavioral management program
}

\begin{abstract}
Introduction. In the present study, the application of a program of handling of contingencies for the improvement of the behavior in a classroom of obligatory Secondary Education is exposed, where the majority of the pupils presented/displayed a disruptive behavior.

Method. For this study a behavioural treatment denominated Good Behavior Time (GBT) was applied, based on a system of "token economy" oriented towards the group with interdependent contingencies, adapted partly of similar techniques like the "Game of Good behaviour Game, (GBG) and the technique of the "clock of good behavior".

Results. As far as the results in obtained "Time of Good behavior", an important improvement is observed clearly that takes to reach $90 \%$ of the time of the school sessions of 50 minutes, from approximated $50 \%$ of the line bases

Discussion y Conclusion: The results of this study show to the effectiveness of the technique of contigencia with groups "Good Behavior Time", (GBT) in the reduction of disruptive behaviors in educative contexts. The qualities of this technique are the facility of their operation as much for the pupils as for the teaching staff, his low economic cost as well as its adaptation to the characteristics of each group, aspects that conform the result of wished effectiveness.
\end{abstract}

Keywords: techniques of group contingencies, good behavior time, (gbt), good behavior game (gbg), disruptive behavior, improvement of the coexistence in the classroom, token economy, clock of good behavior.

Received: 10/01/08 Initial Acceptance: 10/14/08_Definitive Acceptance: 02/24/09 


\section{Introducción}

Los principios y procedimientos psicológicos del Análisis y Modificación de la Conducta aplicados en el control del comportamiento disruptivo y la mejora de la convivencia en el aula, tienen ya un importante acervo científico recogido en una bibliografía empírica que se nos muestra ingente e incluso clásica fuera de nuestras fronteras (Hewett, 1967; McAllister, Stachowick, Baer y Condemab, 1969; O’Leary, Becker, Evans y Saudargas, 1969; Pattterson, Jones Whitier y Wright, 1965; Ward y Baker, 1968) aunque, son todavía escasos los estudios y aplicaciones recogidas en población española.

Estos procedimientos vuelven ahora a primera línea de actualidad debido al preocupante aumento de comportamientos disruptivos en el alumnado escolarizado en los últimos cursos de la actual educación obligatoria, etapa prologada hasta los dieciséis años que ahora abarca más cursos escolares que en épocas anteriores. Cuando hablamos de comportamiento disruptivo en el aula, se entiende toda conducta de inadaptación que dificulte las posibilidades de relación e integración en el contexto educativo así como la adquisición de repertorios funcionales en su aprendizaje formal.

El creciente interés y aumento de Jornadas, Cursos y Congresos dirigidos al profesorado y dedicados al tema de la convivencia en el aula queda plasmado funcionalmente en la abundante proclamación de Reglamentos de Organización y Funcionamiento, así como en normativas específicas a nivel más general que desde el tipo de Instrucciones y Órdenes llega incluso hasta el rango de Decretos, legislados específicamente para mejorar la convivencia en las aulas. Con este mismo objetivo, la Administración educativa está instaurando además, lugares permanentes como páginas web y Observatorios de Convivencia desde donde vertebrar la información y recursos disponibles para favorecer los niveles de convivencia necesarios para llevar a cabo un adecuado proceso de enseñanza-aprendizaje en los centros educativos.

Aunque en la legislación educativa actual se recogen procedimientos derivados de técnicas conductuales como el coste de respuesta, el tiempo fuera o la sobrecorrección, como intervención individual en casos de conductas perjudiciales para la convivencia, a menudo resulta complicado aplicar estos procedimientos en aulas en las que un importante número de alumnado presenta comportamientos disruptivos. En este caso (cada vez más frecuente en el tramo de 12 a 14 años de la escolaridad obligatoria), las técnicas de contingencias orientadas 
hacia el grupo (Tankersley, 1995) se muestran tan efectivas como los procedimientos individuales de intervención antes aludido, con la ventaja añadida de que resultan más fáciles de aplicar por el profesorado e incrementa la cohesión grupal del alumnado en la mejora de comportamientos que posibiliten el adecuado proceso de enseñanza- aprendizaje (Gresham, 1983).

El sistema de contingencias de grupo es aquel programa en el que la realización de conductas previamente establecidas, acarrea la consecución o pérdida de un refuerzo basado en el comportamiento individual de los integrantes del grupo (Albert y Troutman, 1995; Cooper, Heron y Heward, 1987). Las contingencias pueden, a su vez, ser Dependientes, cuando los integrantes del grupo consiguen el refuerzo si un individuo realiza la conducta establecida; Independientes, cuando sólo reciben el refuerzo los miembros del grupo que cumple el criterio comportamental; e Interdependientes, cuando todos los integrantes del grupo reciben refuerzo simultáneamente en función de unos objetivos también grupales (Cooper, et al., 1987).

En esta dirección, los "sistemas de economía de fichas" se muestran como procedimientos idóneos para basar un programa de contingencias grupal que persiga introducir o reorganizar contingencias ambientales a través del control de un estímulo reforzador generalizado, el cual queda previamente establecido y cuya ocurrencia puede controlarse completamente. Como quiera que el reforzador debe presentar una dimensión física y dado que en las primeras aplicaciones de esta técnica se utilizaron fichas de póker, es por ello que quedó definido sustantivamente como "economía de fichas".

\section{Objetivos e hipótesis}

En el presente estudio, se expone la aplicación de un programa de manejo de contingencias para la mejora del comportamiento en un aula donde la mayoría del alumnado presentaba un comportamiento disruptivo que dificultaba en gran medida no solo la labor discente sino también la docente. Para este estudio se aplicó un tratamiento conductual basado en un sistema de "economía de fichas" orientado hacia el grupo con contingencias interdependientes y adaptando en parte la técnica del "reloj de buena conducta" (Barkley, 1995).

La hipótesis de partida es que con el programa "Tiempo de Buena Conducta” (Good behavior Time, GBT) podríamos disminuir la frecuencia de la conducta disruptiva en el aula. 


\section{Método}

\section{Participantes}

La aplicación se llevó a cabo en un grupo- clase de $1^{\text {o }}$ de Educación Secundaria Obligatoria (ESO) compuesto por 26 alumnos, de los cuales 9 eran niñas y 17 niños. Las edades estaban comprendidas entre los doce y trece años, siendo esta última la de mayor prevalencia ya que la mayoría del alumnado de este grupo, había repetido algún curso en la etapa de la Educación Primaria. Aún así no habían conseguido los objetivos mínimos de la etapa de Primaria y por tanto presentaban un desfase de uno o dos cursos en su competencia curricular. Prácticamente todos provenían de un colegio anexo al instituto y pertenecían a una clase de nivel socioeconómico bajo.

\section{Instrumentos y secuencias de evaluación}

Las técnicas utilizadas en la evaluación del caso han sido la entrevista conductual, hetero-observación, registros de tiempo y actas de calificaciones escolares.

a. Evaluación pretratamiento.

- Entrevista semiestructurada con el equipo educativo.

- Entrevista semiestructurada con el tutor.

- Informes de hetero-observación del equipo educativo.

- Informes de hetero-observación del tutor.

- Informes y Expediente académico del curso anterior.

- Registro de línea base de "Tiempo de Buena Conducta" mediante reloj.

b. Evaluación intratramiento.

- "Valoración interjueces" del "Tiempo de Buena Conducta" semanal, mediante reloj.

- Actas de evaluación curricular trimestral.

c. Evaluación resultado del tratamiento.

- Evaluación de objetivos conductuales propuestos.

- Actas finales de evaluación curricular.

d. Evaluación de seguimiento. 
- La evaluación de seguimiento no es posible en este caso debido al carácter contextual irrepetible en cursos siguientes, ya que el agrupamiento se hace en función de las asignaturas optativas e itinerarios curriculares elegidos, amén del alumnado que repite curso.

\section{Análisis Conductual}

1. Análisis de la situación problema.

El alumnado del grupo clase emite mayoritariamente conductas incompatibles con la atención y realización de tareas de aprendizaje escolar.

Análisis de las respuestas en términos topográficos:

- Cognitivas: desmotivación generalizada hacia las tareas escolares. Bajas expectativas de autoeficacia.

- Fisiológicas: nerviosismo e inquietud en el aula.

- Motoras: se muestran impulsivos e hiperactivos en conductas incompatibles con el aprendizaje (charlar en clase, levantarse a menudo del asiento, inatención)

2. Análisis de los estímulos antecedentes.

- Próximos: cambio de etapa educativa de Primaria a Secundaria. Pasar de tener un maestro tutor y dos o tres maestros especialistas en la etapa de Educación Primaria (idiomas, educación física, plástica, y otras) a nueve profesores en $1^{\circ}$ de Secundaria. Más interrupciones y entradas y salidas de profesores en clase y por tanto mayor espacio temporal en el que los alumnos están solos. Mayor exigencia curricular. Pasar, en dos meses, de ser los mayores del colegio a ser los más jóvenes del instituto, etc...

- Remotos: historia de fracaso escolar generalizado. Rechazo y evitación de las tareas escolares sentidas como estímulos aversivos.

3. Análisis de los estímulos consecuentes.

- Próximos: aplicación generalizada de amonestaciones y castigos por parte del profesorado así como calificaciones negativas del aprendizaje. De parte de los compañeros de clase, refuerzo de las conductas disruptivas incompatibles y a veces hasta liderazgo frente al profesorado que se vive como elemento aversivo.

- Remotos: Continuidad del fracaso escolar y sentimiento de inadecuación generalizado en la institución educativa como alumno. Fracaso Escolar. 
4. Análisis de autocontrol.

Ante las bajas expectativas de autoeficacia en su aprendizaje, la mayoría del alumnado de este grupo muestra conductas de escape y evitación ante las tareas escolares.

5. Recursos de los sujetos.

Las conductas de atención son cortas y pocas. Se tiende a evitar tareas que impliquen un esfuerzo cognitivo.

6. Factores determinantes en la adquisición de la conducta problema.

\section{Estímulo}

Tareas docentes y discentes de aprendizaje curricular.

Manifestaciones orgánicas

Nerviosismo, inquietud, expectativas de fracaso.

\section{Respuesta}

Rechazo de las actividades implicadas en el aprendizaje formal. Evitación con conductas incompatibles e incluso distintas modalidades de escape.

\section{Consecuencia}

El profesorado amonesta al alumnado y en vista de la reiteración de conductas contrarias a la convivencia se les aplica la técnica del "tiempo fuera" y se les expulsa de clase e incluso cuando son reiteradas las conductas disruptivas, fuera del instituto durante algunos días.

\section{Contingencia}

Los alumnos al ser expulsados de clase se atienden en un aula de trabajo donde coinciden varios, lo cual les sirve doblemente de reforzador: por un lado dejan atrás la presión y esfuerzo cognitivo que se exige en una sesión docente de clase (refuerzo negativo) y por otro (como refuerzo positivo) se reúnen varios compañeros convirtiéndose ésta en lugar de consecución de un reforzador social entre los iguales.

Cuando son expulsados del Centro algunos días, por reiteración de faltas, a la mayoría les falta un control familiar y suelen estar en la calle, relajados y divirtiéndose con otros del barrio en igual situación. Incluso si no es así, quedan en casa frente a la televisión o los videojuegos. 


\section{Análisis funcional}

El alumnado de este grupo de $1^{\circ}$ de ESO presentaba mayoritariamente un alto nivel de conductas disruptivas incompatibles con la labor discente y docente. El cambio de etapa educativa en la que encuentran un equipo de profesores de más del doble que en los últimos curso de Primaria acarrea a menudo mayor dificultad de seguimiento y coordinación y por tanto una mayor exigencia de autocontrol de alumnado. A este factor hay que unir la dificultad de autorregulación de la atención y conducta de este grupo de alumnos que presentaba una historia de fracaso escolar importante, origen de una nula disposición y alta desmotivación hacia las tareas escolares acompañada de una negativa imagen de sí mismos como estudiantes así como bajas expectativas de logro escolar en el futuro.

La motivación intrínseca del aprendizaje por el aprendizaje es una actitud cognitiva ausente en este alumnado que incluso plantea también desinterés e incluso rechazo, por otras motivaciones extrínsecas como pueden ser las calificaciones escolares. Descartadas las calificaciones del aprendizaje como fuente de refuerzo y planteándose más bien como estímulos aversivos o neutros, al alumnado solo le queda el refuerzo social de sus iguales mediante actividades lúdicas y de entretenimiento dentro del aula y ajenas e incompatibles con cualquier situación de aprendizaje formal, refuerzo social que continúa en el aula de trabajo si coinciden varios alumnos expulsados y se dedican a charlar entre ellos e incluso si son expulsados por unos días del Centro se lo toman como un privilegio vacacional compartidos con otros en la misma situación o en casa viendo la televisión o entreteniéndose con los videojuegos, actuando por tanto las contingencias como refuerzo positivo a las conductas disruptivas, combinado con el refuerzo negativo de evitar la situación de presión y esfuerzo cognitivo del aula.

\section{Procedimiento}

Dos meses después de comenzar el curso escolar y en el contexto de una reunión de preevaluación de un grupo-clase de $1^{\circ}$ de ESO, se pone de relieve de manera generalizada por parte del equipo educativo de este grupo, el desfase curricular que presenta el alumnado referido así como la baja motivación hacia las tareas de aprendizaje y el alto nivel de desatención que presentan acompañado de conductas disruptivas. 
Se inicia por parte del psicólogo del departamento de Orientación del Centro, en un primer momento y como técnica de identificación del problema, una entrevista conductual y grupal con un carácter abierto para dar cauce a la necesidad de expresión y desahogo que se constata entre el profesorado. En esta entrevista se extrae información en líneas generales, relativa a la naturaleza del problema, la importancia, así como la frecuencia con que se da.

El profesorado expone en esta entrevista grupal que lo que más le preocupa es el inadecuado comportamiento de este alumnado ya que está impidiendo la aplicación de medidas de adaptación curricular, las cuales ya están elaboradas y preparadas para cuando el alumnado lo permita y se pueda "dar la clase en condiciones adecuadas".

Posteriormente se lleva a cabo una entrevista individual entre el psicólogo y el tutor del grupo, en la que se le propone el programa de tratamiento conductual para "disminuir las conductas disruptivas y aumentar la conducta de atención por parte del alumnado". Esta entrevista tiene como finalidad devolverle información al tutor, referido al problema que presenta su grupo, desde el punto de vista del Análisis Funcional de la Conducta así como las líneas generales del programa terapéutico grupal propuesto.

Posteriormente se lleva a cabo otra entrevista grupal con el equipo educativo, en la que una vez que se cuenta con la aprobación del tutor se devuelve información al profesorado al igual que se hizo primeramente con el tutor del grupo. Además, se les da la información pertinente para que puedan medir "una línea base" del tiempo que realmente se puede dar clase en cada sesión lectiva de cada materia. Se buscó por parte del psicólogo, presentar un programa de intervención fácil de comprender y de realizar por el equipo docente compuesto por 11 profesores, de tal manera que facilitará la adherencia al programa así como buenas expectativas hacia la eficacia de éste. Tan solo dos profesores no se sumaron al programa.

El proceso de evaluación no termina en esta primera fase de consecución de datos, sino que continúa durante la fase de tratamiento con el objetivo de evaluar la evolución de las conductas objetivos desde la primaria línea base.

Para tal fin, una vez comenzado el programa se evaluó "el Tiempo de Buena Conducta" (Good behavior Time, GBT) por parte del profesorado, utilizando un cronómetro que entregaba el delegado del grupo clase al comenzar cada sesión lectiva. Durante ésta, el profeso- 
rado ponía el cronómetro en marcha cuando el alumnado mostraba las conductas criterio definidas previamente entre el psicólogo escolar y el tutor. Cuando el docente estimaba que no se estaban cumpliendo los criterios de refuerzo se lo hacía saber verbalmente al alumnado y paraba el cronómetro hasta que volvían a advertirse las conductas deseadas.

Al finalizar cada sesión lectiva el docente anotaba, en un registro que le facilitaba el delegado (o en su ausencia el subdelegado) de clase, el tiempo total acumulado en su sesión y lo firmaba. Semanalmente en la hora de tutoría, se evaluaba el tiempo conseguido (cuya puntuación hacía los efectos de reforzador generalizado) para en función de éste asignar y cambiarlos por refuerzos concretos al grupo- clase.

A lo largo del programa se utilizan las sesiones semanales de coordinación del tutor con el psicólogo escolar para evaluar los tiempos conseguidos y proponer las siguientes marcas de tiempo semanales a conseguir por el alumnado. Como el tiempo valorado conseguido era la suma de las distintas sesiones lectivas del equipo de profesorado de este curso, lo que realmente se valoraba era una puntuación ponderada que evitaba y compensaba las distintas subjetividades a la hora de considerar conseguidas las conductas criterio. Se buscaba conseguir un tipo de "valoración interjueces" ponderada.

El grado de aumento de "Tiempo de Buena Conducta" general fue el índice primario considerado finalmente para evaluar la eficacia del programa. También se evaluó el porcentaje, mediante las medias aritméticas, de calificaciones escolares del grupo a lo largo del curso, como medida secundaria significativa y funcional de la utilidad del programa.

\section{Tratamiento}

La Metodología del programa conductual está basado en un sistema de "economía de fichas" orientado hacia el grupo, aplicando contingencias interdependientes y adaptando en parte la técnica del "reloj de buena conducta" (Barkley, 1995). Más concretamente, está dirigido a reforzar al alumnado por comportamientos escolares adecuados, con actividades y gratificaciones cercanas a su contexto vivencial. Como el refuerzo es siempre social y de su posible consecución son responsables los miembros del grupo, esta relación causal exige mecanismos de autorregulación grupal donde el mismo alumnado actúa de agente de cambio de sus compañeros de clase. Estas contingencias de grupo estaban acompañadas de los componentes 
individuales que en la legislación actual se recoge como intervención individual en casos de conductas perjudiciales para la convivencia y que consisten básicamente, como ya se ha comentado anteriormente, en procedimientos derivados de técnicas conductuales como el coste de respuesta, el tiempo fuera o la sobrecorrección.

En este programa de contigencia grupal, el alumnado debía conseguir "tiempos de buena conducta" en las distintas asignaturas (9) en las que se aplicaba el programa. Al principio conseguir un 50\% del horario, para que el alumnado no dejara de emitir comportamientos adecuados una vez que calcularan que habían conseguido los objetivos, se instauraron otros premios cuyo logro iba supeditado a la consecución sumativa de objetivos por encima del mínimo estimable, tal como se recoge más adelante en el punto dedicado al establecimiento del programa.

Se definió como "tiempo de buena conducta":

- Atender al profesor.

- Permanecer sentados.

- Escuchar al profesor y a los compañeros cuando lo autorice el profesor.

- Pedir la palabra cuando se quiera decir algo.

- Realizar las actividades.

Este tiempo se medía por cada profesor/a, utilizando un cronómetro que le era entregado por un alumno encargado, al comenzar cada clase. Durante cada sesión lectiva, el discente ponía el cronómetro en marcha cuando los alumnos mostraban las conductas adecuadas. Cuando el profesor/a estimaba que no se estaban cumpliendo los criterios conductuales se lo hacía saber al alumnado y detenía el cronómetro hasta que volvían a evidenciarse las conductas deseadas. Al final de clase el discente anotaba en un registro que le aportaba el alumno encargado, el tiempo total acumulado hasta el final de su clase y lo firmaba. Semanalmente el tutor revisaba los tiempos conseguidos y reportaba al alumnado los premios conseguidos. No se tenían en cuenta en el cómputo semanal, el tiempo en los que faltaba algún profesor.

Si algún alumno era expulsado de clase, se restaba 5 minutos al tiempo de buena conducta conseguido por el grupo. Este tiempo de coste de respuesta se fue aumentando a lo largo del programa. Los premios los propuso el alumnado en sesión de tutoría. Los votaron y en función de su mayor valor reforzante, el tutor los ordenó de menor a mayor y los graduó de esta forma en relación a los tiempos que habían de conseguir. 


\section{Información al Equipo Educativo.}

Mediante reuniones de coordinación del equipo educativo con el orientador y psicólogo escolar del Centro se hace explícito el problema y la necesidad de intervención y remediación. Una vez recogida la información académica y conductual necesaria para el análisis funcional de la conducta en clase del grupo, se devuelve al equipo educativo, también en reunión de coordinación, la información y el análisis funcional de la conducta disruptiva grupal así como instrucciones para recoger la "línea base" inicial del programa de intervención. Una vez establecida la "línea base", un resumen de esta información queda plasmada explícitamente en un documento informativo que se proporciona a cada discente (anexo I).

\section{Establecimiento de la Línea Base}

La línea base fue considerada y consensuada con el equipo educativo como el tiempo efectivo de clase ya que gran parte del tiempo lectivo, el profesorado se tenía que dedicarse a mandar a callar y a reprobar conductas disruptivas. Se estimó en el 50\% de cada sesión lectiva, o sea 25 minutos. Se partió de los niveles de atención que el alumnado estaba ya ejecutando para favorecer en un primer momento del programa, la consecución de reforzadores y por tanto la adhesión al programa.

\section{Información al alumnado del grupo y determinación de los Reforzadores}

En el procedimiento para establecer los reforzadores se utilizó el "Principio de Premack", es decir el uso de actividades o privilegios para efectuar actividades muy deseadas por el alumnado que actúan como reforzadores de actividad mucho más económico, fácil e incluso sociable que los reforzadores materiales.

Para que los reforzadores tuviesen valor para los sujetos, se decidió que fueran propuestos por los estudiantes. De esta manera se dedicó una sesión de tutoría para que el alumnado propusiera, siguiendo la técnica del "torbellino de ideas", para posteriormente pasarlos a votación y en función de su mayor valor reforzante, el tutor los ordenara de menor a mayor y los graduara de esta forma en relación a los tiempos que había de conseguir el alumnado. Se les indicó al alumnado que no se consideraban reforzadores que supusieran un coste oneroso para el Instituto. 
Para hacernos una idea del tipo de reforzador que propuso y votó el alumnado podemos citar los siguientes: bajar al patio con el profesor de guardia en hora libre, utilizar los ordenadores también en horas libres, cinco minutos libres al final de la clase antes del recreo, invitación a un desayuno, ver películas en horas libres cuando falta algún profesor, escuchar música de fondo en clase, ir de excursión, etc...

La lista de premios se dividió en tres partes, la primera incluía aquellos refuerzos que habían sido menos votados (más baratos), la segunda parte los que recibieron la mayor parte de votos (más caros) y la parte intermedia formada por premios medianos (precio de mercado). Para aceptar una propuesta como premio, se estipuló que debía conseguir al menos un $60 \%$ de los votos.

Asimismo se les informó que si algún alumno era expulsado de clase, se restaba 5 minutos al tiempo de buena conducta conseguido por el grupo. Este tiempo de "coste de respuesta" se fue aumentando a lo largo del programa. El objetivo de esta medida era que el grupo influyera positivamente en los alumnos más disruptivos para mejorar su comportamiento o que al menos, no fuera reforzado por el grupo.

\section{Establecimiento del Programa}

Una vez comenzado el programa cada docente media "el Tiempo de Buena Conducta" (Good behavior Time, GBT) en sus clases lectivas, utilizando un cronómetro que le entregaba el delegado del grupo clase al comenzar cada sesión lectiva. Durante ésta, el docente ponía el cronómetro en marcha cuando el alumnado mostraba las conductas criterio definidas previamente entre el psicólogo escolar y el tutor.

Las conductas que era prioritario controlar y eliminar en el aula, se reestructuraron para definirlas en positivo. De esta manera, además de renunciar a un carácter punitivo de la modificación de las conductas disruptivas, se ponía el énfasis en el desarrollo de patrones adecuados de conductas. De esta manera, se definen claramente unas habilidades y repertorios conductuales las cuales en su desarrollo son incompatibles con las conductas problema a eliminar.

En cuanto al número de éstas se determinaron cinco marcas, al igual que otro tipo de técnicas parecidas como el "Juego del Buen Comportamiento" (Good behavior Game, GBG) 
desarrollado por Barrish, Saunders y Wolf (1969). En nuestro caso fueron las siguientes: atender al profesor, permanecer sentados, escuchar a los compañeros cuando éstos expongan o pregunten algo en clase, pedir la palabra cuando se quiera decir algo y realizar en clase las tareas que se determine.

Semanalmente se fue evaluando en tutoría la consecución de las conductas objetivo del programa, las cuales una vez conseguidas con el mínimo estipulado facilitaban el conseguir un premio barato. Si superaban el mínimo de tiempo semanal en un $25 \%$ conseguían otro premio adicional (barato). Los refuerzos se los proporciona el tutor en su hora de tutoría. Los premios se iban alternando para no producir saciedad. Cada mes en la tutoría se trabajaban la propuesta de nuevos premios y su valoración (votación) que se comparaba con la primera lista para darle el valor correspondiente. Mensualmente se revisó la consecución de objetivos. Si se habían conseguido (aunque sea el mínimo estipulado) en tres semanas consecutivas, conseguían un premio adicional de los medios (mercado). Si se conseguían en cuatro semanas podían obtener un premio alto (caros). Cuando conseguían los objetivos dos semanas seguidas, se aumentó la exigencia de "tiempo bueno" en la cantidad que se estimaba conveniente en función de los resultados conseguidos.

\section{Resultados}

\section{Evaluación de resultados conductuales}

A continuación, primero en la tabla 1 y después en la figura 1 , se presentan los criterios de refuerzo establecidos en cada una de las 14 semanas del programa, así como el "tiempo de buena conducta" conseguido y los refuerzos contingentes que se aplicaron.

\section{Evaluación de resultados académicos}

Aunque el objetivo del programa "Tiempo de Buena Conducta" (Good behavior Time, $G B T)$ es disminuir la frecuencia de la conducta disruptiva en el aula, también se evaluaron los resultados de aprendizaje del segundo y tercer trimestres con los conseguidos antes de aplicar el programa, en el primer trimestre. Los datos obtenidos reflejan un descenso (figura 2) en el porcentaje de suspensos que básicamente permanece hasta final de curso. Se puede observar 
fácilmente la mejoría de resultados comparando los obtenidos por el grupo en el primer trimestre con los de finales de curso.

Tabla 1. Criterios de refuerzo establecidos en cada semana, así como el "tiempo de buena conducta" conseguido y los refuerzos contingentes que se aplicaron.

\begin{tabular}{|c|c|c|c|}
\hline SEMANAS & $\begin{array}{c}\text { CRITERIO } \\
\text { REFUERZO }\end{array}$ & $\begin{array}{c}\text { TIEMPO } \\
\text { OBTENIDO }\end{array}$ & REFUERZOS \\
\hline $1^{\mathrm{a}}(1-2)$ & $25^{\prime}$ & $35^{\prime}$ & Música de fondo \\
\hline $2^{a}(2-2)$ & $25^{\prime}$ & $33^{\prime}$ & $\begin{array}{c}\text { Desayuno en el ins- } \\
\text { tituto (gratis) }\end{array}$ \\
\hline $3^{a}(3-2)$ & $25^{\prime}$ & $38^{\prime}$ & Informática \\
\hline $4^{a}(4-2)$ & $40^{\prime}$ & $41^{\prime}$ & $\begin{array}{l}\text { Desayuno (gratis) } \\
\text { fuera del instituto }\end{array}$ \\
\hline $5^{a}(1-3)$ & $40^{\prime}$ & $41^{\prime}$ & $\begin{array}{c}\text { Sin refuerzo (por } \\
\text { coste de respuesta } \\
\text { expulsión) }\end{array}$ \\
\hline $6^{a}(2-3)$ & $40^{\prime}$ & $35^{\prime}$ & Sin refuerzo \\
\hline $7^{a}(3-3)$ & & & $\begin{array}{c}\text { Vacaciones por Se- } \\
\text { mana Santa }\end{array}$ \\
\hline $8^{a}(4-3)$ & $40^{\prime}$ & $35^{\prime}$ & Sin refuerzo \\
\hline $9^{a}(1-4)$ & $40^{\prime}$ & $35^{\prime}$ & Sin refuerzo \\
\hline $10^{\mathrm{a}}(2-4)$ & & & $\begin{array}{c}\text { Vacaciones por } \\
\text { Fiestas }\end{array}$ \\
\hline $11^{a}(3-4)$ & $35^{\prime}$ & $37^{\prime}$ & $\begin{array}{c}\text { Salir al Patio en } \\
\text { horario de inglés } \\
\text { (asignatura del tu- } \\
\text { tor) }\end{array}$ \\
\hline $12^{a}(4-4)$ & $35^{\prime}$ & $41^{\prime}$ & Uso de internet \\
\hline $13^{a}(1-5)$ & $35^{\prime}$ & $45^{\prime}$ & $\begin{array}{c}\text { Patio en hora de } \\
\text { tutoría }\end{array}$ \\
\hline $14^{a}(2-5)$ & $35^{\prime}$ & $47^{\prime}$ & Concurso en clase \\
\hline
\end{tabular}




\section{Minutos}

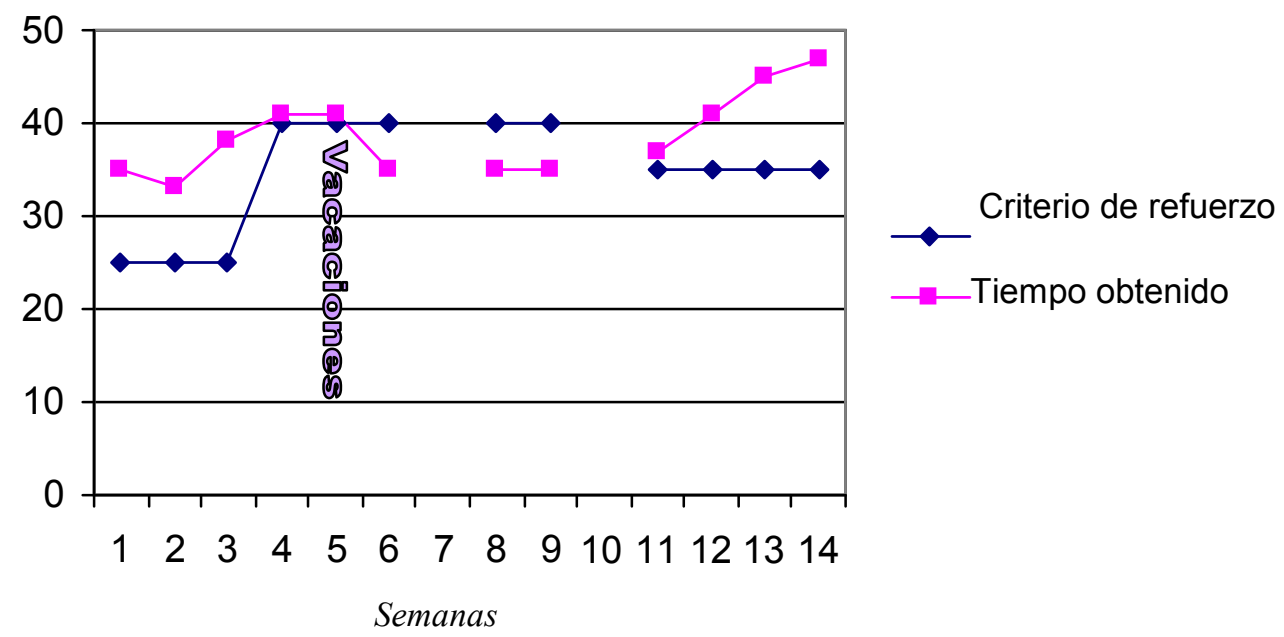

Figura 1. Evolución de los criterios de refuerzo y consecución de "Tiempo de Buena Conducta" (Good behavior Time, GBT)

\section{$\square$ 1er trim. $\square$ 2do trim. $\square$ 3er trim.}

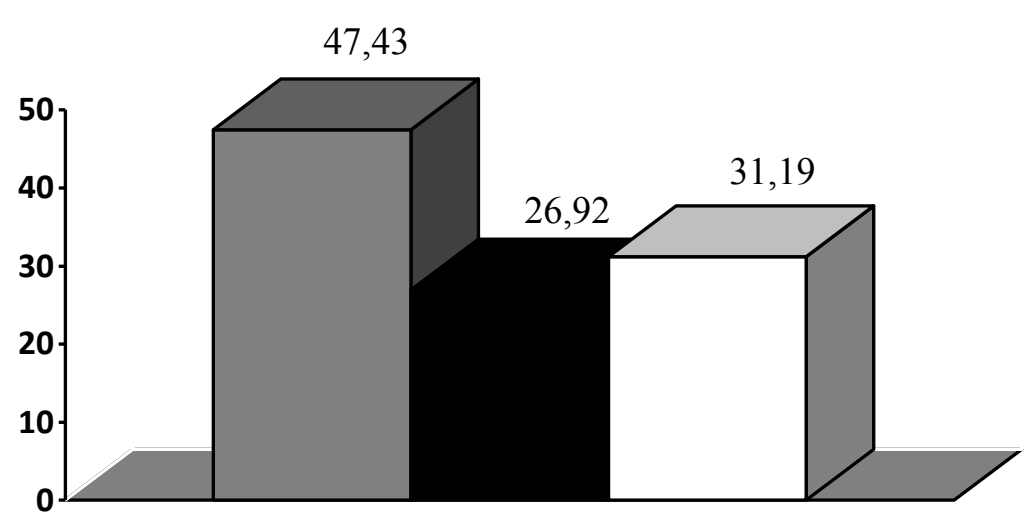

Figura 2. Porcentaje de suspensos por trimestres de las asignaturas en las que se ha llevado a cabo el Programa "Tiempo de Buena Conducta" (Good behavior Time, GBT)

Para comparar resultados se midieron asimismo los porcentajes de suspensos en las dos asignaturas donde no se aplicó el programa. Se observa en la figura 3, una mejora de resultados que puede ser en parte atribuida a una generalización de los efectos positivos del programa GBT, aunque se aprecia (figura 4) un menor índice de mejoría que en las asignaturas en las que se aplicó directamente. Mientras el grupo de asignaturas donde se aplicó el pro- 
grama GBT, mejoró sus resultados académicos en un 34,23\% mientras que en el grupo que no se aplicó tan solo lo hizo en un 15,61\%.

\section{$\square$ 1er trim. $\square$ 2do trim. $\square$ 3er trim.}

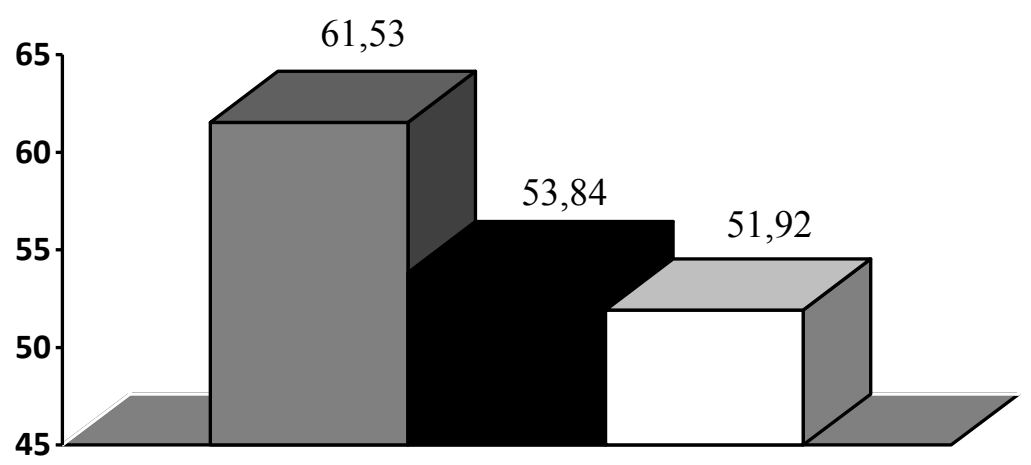

Figura 3. Porcentaje de suspensos por trimestres de las asignaturas en las que se no se ha llevado a cabo el Programa de Modificación de Conducta

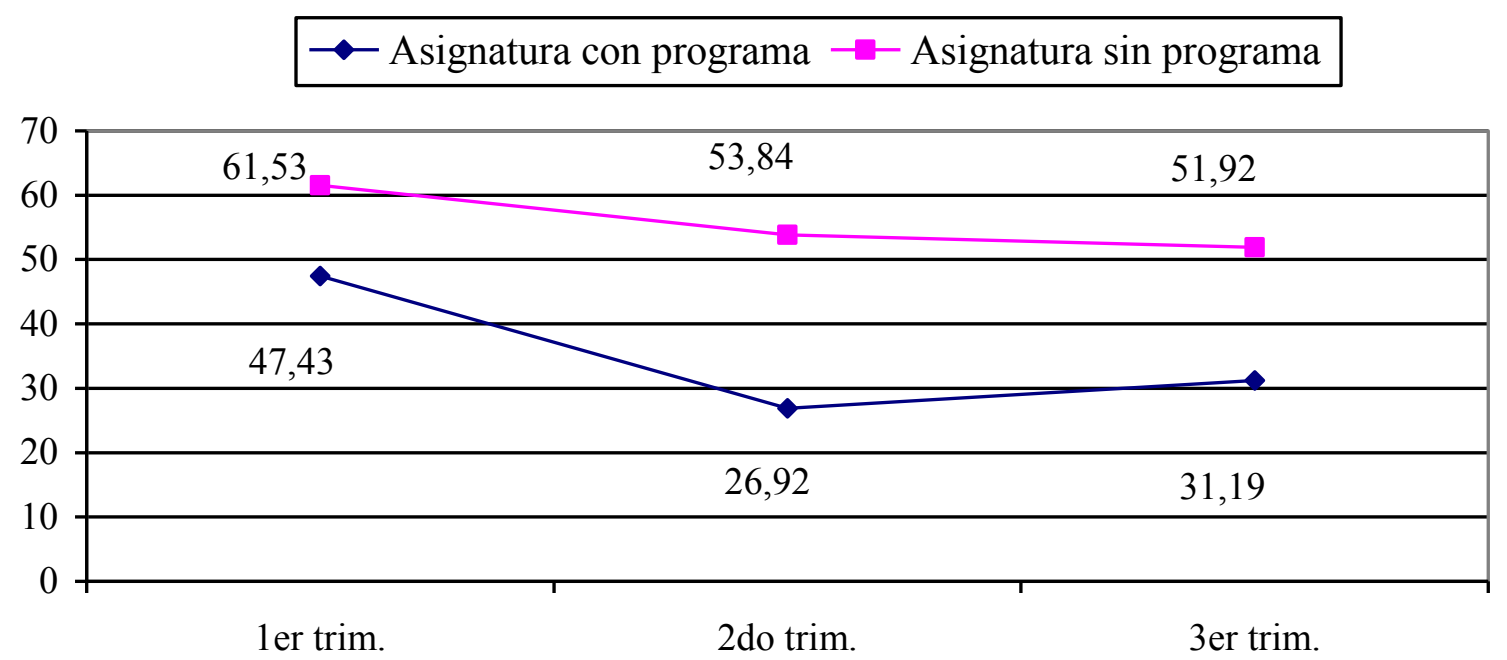

Figura 4. Comparación de resultados académicos según las asignaturas con y sin Programa.

\section{Conclusiones}

Los resultados de este estudio muestran la eficacia de la técnica de contigencia con grupos "Tiempo de Buena Conducta" (Good behavior Time, GBT) en la reducción de comportamientos disruptivos en contextos educativos. Las cualidades de esta técnica son la facili- 
dad de su funcionamiento tanto para el alumnado como para el profesorado, su bajo coste económico así como su adaptación a las características de cada grupo, aspectos que conforman el resultado de eficacia deseada.

Como quiera que la reducción en la frecuencia y cantidad de comportamiento disruptivo favorece el proceso de enseñanza aprendizaje, también se buscó como efecto positivo secundario la mejora de los aprendizajes escolares. Con esa finalidad, el programa de intervención conductual se complementó con la elaboración de adaptaciones curriculares de aula por parte del equipo educativo, que aunque estaban elaboradas no podían llevarse a cabo debido a la frecuencia de comportamientos disruptivos incompatibles con el proceso de enseñanza aprendizaje.

En cuanto a los resultados en " "Tiempo de Buena Conducta" obtenidos, se observa claramente una importante mejora que lleva a alcanzar el 90\% del tiempo de las sesiones lectivas de 50 minutos, desde el 50\% aproximado de la línea base. Por tanto, el objetivo propuesto se consiguió con creces.

Se midieron también los resultados de aprendizaje disciplinar, para ver si secundariamente al objetivo principal se había conseguido otros objetivos positivos colaterales. En este sentido hay que decir que aunque los resultados de mejora académica, siendo positivos, no lo han sido tanto como los conductuales, es fácil entender como ya se ha comentado, que al disminuir la frecuencia de comportamientos disruptivos incompatibles con el proceso de enseñanza aprendizaje, éste se vea favorecido y facilitado. De todas maneras, es preciso contemplar que el control conductual es mucho más directo que la asimilación y desarrollo de habilidades y competencias académicas, proceso en el que intervienen múltiples factores discentes y docentes.

La medida de la generalización de los beneficios conductuales en los siguientes cursos escolares es difícil en cuanto que la configuración de los grupos tanto de alumnado como de profesorado difieren de un curso escolar a otro.

En cuanto a la generalización de los beneficios secundarios obtenidos en las asignaturas en las que el profesorado aplicó la técnica, se puede suponer que la mejora aunque menor (justamente la mitad), producida también en los resultados escolares de las dos asignaturas en 
las que no se siguió el procedimiento, puede significar una relación de contingencia positiva, en la línea de otros estudios sobre técnicas parecidas (Grandy, Madsen, y De Mersseman, 1973).

En una intervención sistémica como ésta, la adhesión al procedimiento por parte del alumnado conlleva una autorregulación grupal como ya se ha comentado, pero en el subsistema del profesorado es posible encontrar algunas reticencias como ya se ha señalado en otros estudios con técnicas parecidas (Embry, 2002). En estos casos y una vez agotada la explicación funcional y utilidad de la técnica, es conveniente ponerla en marcha con el profesorado comprometido y dejar la puerta abierta a la colaboración de todos, una vez se observen los primeros resultados positivos.

La explicación y fundamentación de la técnica al equipo educativo, para su deseada adhesión, pasa por resaltar que esta intervención tiene como objetivo el desarrollo de competencias y habilidades sociales y motivacionales por parte del alumnado, ya que aunque la técnica se basa en factores motivacionales extrínsecos al proceso de enseñanza- aprendizaje, se sustenta en la utilización de privilegios para llevar a cabo actividades muy deseadas por el alumnado que funcionan como reforzadores de actividad mucho más económicos, fáciles e incluso sociables que otros reforzadores materiales. De esta manera, a la vez que se mejoran las habilidades sociales grupales, se facilita desde factores motivacionales extrínsecos al aprendizaje, la posibilidad de mejores auto-expectativas de logro académico en el alumnado que sustenten la base necesaria para desarrollar en la medida de lo posible, la motivación intrínseca hacia el aprendizaje.

En otra dirección, puede ser incluso que los tutores de otros grupos expresen que su alumnado quiere funcionar del mismo modo. En tal caso no hay ningún impedimento en generalizar su aplicación, más bien sería un buen indicio de la aceptación del procedimiento por parte de los sujetos, con lo que estaríamos asegurando la mejor base para que formara parte, si se estima necesario, del Reglamento de Funcionamiento del Centro o incluso del Plan de Convivencia de éste.

Para finalizar, concluir que un procedimiento de contingencia grupal interdependiente, como la técnica del "Tiempo de Buena Conducta" (Good behavior Time, GBT) puede ser un procedimiento fácil y efectivo para reducir comportamientos disruptivos en el aula, incompa- 
tibles con el proceso de enseñanza- aprendizaje. En esta dirección, este estudio desarrollado en un grupo de alumnado de Educación Secundaria Obligatoria Español, pretende actuar como facilitador de una línea de investigación que se nos presenta bastante prometedora.

\section{Referencias}

Albert, P.A. y Troutman, A.C. (1995). Aplied Behavior Analysis for Teachers. Englewood: Prentice-Hall

Barkley, R. A. (1995). Linkages between attention and executive function. In G. R. Lyon \& N. A. Krasnegor (Eds.), Attention, memory, and executive function. Baltimore, MD: Brookes Publishing Co.

Barrish, H., Saunders, M y Wolf, M. (1969). Good Behavior Game: Effects of individual contingencies for group consequences on disruptive behaviour in a classroom. Journal of applied Behavior Analysis, 2,119- 124.

Cooper, J.O., Heron, T.E. y Heward, W.L. (1987). Aplied Behavior Analysis. Nueva York: MacMillan.

Embry, D. (2002). The pax acts games solution: applying replicated research and current evaluation from the Good Behavior Game for achievement and prevention in schools. Paradigms, 1, 1-20. (http:// www.paxis.org)

Grandy, G., Madsen, C. y De Mersseman, L. (1973). The effects of individual and interdependent contingencias on inapropiate classroom behavior. Psychology in the Schools, 10 (4), 488-493.

Gresham, F. M. (1983). Social skills training with handicapped children: A review. Review of Educational Research, 51, 139- 176.

Hewett, F (1967). Educational engineering with emotionally disturbed children. Exceptional Children, 34, 459- 467.

McAllister, L. W., Stachowiak, J. G., Baer, D. M., and Conderman, L. (1969). The applica tion of operant conditioning techniques in a secondary school classroom. Journal of Applied Behavior Analysis, 2(4), 277-285.

O’Leary, K. D., Becker, W.C., Evans, M.B. y Saudargas, R.A. (1969). A token reinforcment program in a public school: a replication and systematic analysis. Journal of Applied Behavior Analysis, 2, 3- 13

Patterson Gr, Jones, R., Whittier, J., Wright, M.A. (1965). A behaviour modification technique for the hyperactive child. Behaviour Research and Therapy, 3, 217-226. 
Tankersley, M. (1995). A group- oriented contingency management program. Preventing School Failure, 40 (1), 1- 7.

Ward, M. H. and Baker, B. L. (1968). Reinforcement therapy in the classroom. Journal of Applied Behavior Analysis, 1(4), 323-328. 


\section{ANEXOS}

\section{(Anexo I) Información al Equipo Educativo}

\section{Introducción:}

A veces nos encontramos que un importante grupo de alumnado de una clase y nivel determinado, arrastra una historia de fracaso escolar origen de una nula disposición y desmotivación hacia las tareas escolares acompañada de una negativa imagen de sí mismos y ninguna auto-expectativa hacia el futuro.

Desde luego, las calificaciones escolares es quizás el factor que menos logre motivarles (ya que no tienen expectativas de conseguir nada mediante su escolarización) e incluso un porcentaje importante de suspensos pueden producir un efecto de "rebote" y de rechazo aún mayor hacia las materias e incluso profesorado.

Cuando el alumnado "tira la toalla" en su proceso de aprendizaje, no le queda más que pensar a que dedicar el tiempo de clase, habiendo descartado por supuesto cualquier intento de aprendizaje.

Llegado este estado de la cuestión, es necesario un programa consensuado y coordinado del equipo educativo con los objetivos que a continuación se recogen.

La metodología de este programa pasa por premiar a los alumnos por comportamientos escolares adecuados, con actividades y otras gratificaciones mucho más cercanas vivencial y temporalmente que las meras calificaciones escolares. Como la gratificación es siempre grupal y de su posible consecución es responsable el grupo como tal, esto lleva a mecanismos de autorregulación donde los mismos alumnos actúan de agente de cambio de sus compañeros. De esta forma, se pueden conseguir en el alumnado objetivos de conducta mínimos para un clima de aprendizaje básico.

\section{Objetivos:}

- Desarrollar conductas de atención.

- Aumentar la motivación hacia las tareas escolares.

- Mejorar los resultados del proceso de enseñanza- aprendizaje.

\section{Metodología:}


Los alumnos deberán conseguir "tiempos de buena conducta" en las distintas materias. Para comenzar con éxito esta tarea se le propondrá al principio conseguir un 50\% del horario lectivo, para ir aumentando progresivamente a lo largo del desarrollo del programa durante el curso escolar.

Se entiende como "tiempo de buena conducta":

1. Atender al profesor.

2. Permanecer sentados.

3. Escuchar al profesor y a los compañeros cuando éstos tengan autorización para hacerlo.

4. Pedir la palabra cuando se quiera decir algo.

5. Realizar las actividades.

Este tiempo se medirá por cada profesor/a, utilizando un cronómetro que le será entregado por un alumno encargado, al comenzar cada clase. Durante cada sesión lectiva, el profesor/a pondrá el cronómetro en marcha cuando los alumnos muestren las conductas adecuadas. Cuando el profesor/a estime que no se están cumpliendo se lo hará saber al alumnado y parará el cronómetro hasta que vuelvan a emitirse las conductas deseadas. Al final de clase el profesor anotará en un registro que le aportará el alumno encargado, el tiempo total acumulado hasta el final de su clase y lo firmará. Semanalmente el tutor revisará los tiempos conseguidos y reportará a los alumnos los premios conseguidos.

Es fundamental la coordinación y homologación de pautas a seguir desde el equipo educativo para que cualquier planteamiento educativo pueda ser eficaz. 